\title{
Assessment of imaging, pathoanatomy and terminology in posterior tibial tendon dysfunction
}

\author{
Matthew Workman ${ }^{1,2}$ (D), Nick Saragas ${ }^{1,2}$ (D), Paulo Ferra01,2(D) \\ 1. Netcare Linksfield Clinic; Johannesburg, Gauteng, South Africa. \\ 2. Department of Orthopedic Surgery, University of the Witwatersrand, Johannesburg, South Africa.
}

\begin{abstract}
Objective: This study aimed to determine damage/change occurring in the posterior tibial tendon of patients undergoing surgery for posterior tibial tendon dysfunction (PTTD) and to correlate preoperative imaging and intraoperative findings with histology to determine the most appropriate investigations for diagnosis. The secondary aim was to clarify terminology used in describing the tendon pathology, to improve descriptive terminology for research, assessment, and treatment of PTTD.

Methods: The records of patients who had undergone surgery for stage 2 PTTD were retrospectively reviewed. Cases in which preoperative diagnostic imaging was done and a posterior tibial tendon specimen was sent for histology were included. Ultrasound (US) and MRI findings, surgical notes and histopathological reports were evaluated.

Results: Nineteen patients met the inclusion criteria. Fourteen had US showing degenerative changes and synovitis. Five had MRI showing tendon degeneration, with rupture in two cases. Intraoperatively, all tendons showed gross abnormality, with surrounding synovitis. Microscopically, no acute inflammation was noted within any tendon specimens. All had non-specific reactive changes within the visceral synovium.

Conclusion: This study confirms clear histological degeneration within the posterior tibial tendon of patients undergoing corrective surgery for PTTD. Preoperative imaging and surgical findings identified tendon sheath synovitis. Pre-operative ultrasound imaging and intraoperative confirmation of PTTD is accurate; thus, histological confirmation is unnecessary. The pathological changes in PTTD have been described as a tendinopathy in the literature. We suggest using the term pantendinopathy, which is a combination of peritendinitis with tendinosis, as it better describes the pathological process.
\end{abstract}

Level of Evidence IV; Therapeutic Studies; Case Series.

Keywords: Posterior tibial tendon dysfunction/surgery; Posterior tibial tendon dysfunction/pathology; Diagnostic imaging; Tendinopathy.

\section{Introduction}

Adult acquired flatfoot deformity (AAFD) is a common disorder characterised by progressive collapse of the medial longitudinal arch, valgus deformity of the hindfoot and forefoot abduction and supination ${ }^{(1,2)}$. Posterior tibial tendon dysfunction (PTTD) is thought to be the most common cause in adults. The aetiology of PTTD is multifactorial and includes abnormal loading of the arch (commonly seen in middleaged obese women), inflammatory disorders, and trauma. Risks factors such as hypertension or diabetes can also be present. The damage often occurs in a zone of hypovascularity, $2-6 \mathrm{~cm}$ from the navicular insertion site ${ }^{(3)}$.
PTTD was classified by Johnson and Strom ${ }^{(4)}$ in 1989, and subsequently modified by Myerson ${ }^{(5)}$ into four stages. Stage Il disease is characterised by enlargement and elongation of the posterior tibial tendon (PTT), rendering it functionally incompetent. This results in a pes planovalgus. Failing initial conservative management, surgical treatment of PTTD consists of joint-sparing surgery, including osteotomies and tendon transfers to rebalance the foot, or fusions ${ }^{(6)}$.

Initial imaging in PTTD consists of weightbearing x-rays, which allow evaluation of bony alignment, osseous changes, and joint arthrosis. Ultrasound (US) and/or MRI assist in making a diagnosis of PTTD. US is preferable and is reported
Work performed at the Netcare Linksfield Clinic; Johannesburg, Gauteng, South Africa.

Correspondence: Matthew Workman. 24 Twelve Av., Linksfield West, Johannesburg, Gauteng, South Africa, Zip Code: 2192. E-mail: matthew.workman1@gmail.com Conflicts of interest: none. Source of funding: none. Date received: June 21, 2020. Date accepted: July 14, 2020. Online: August 30, 2020.

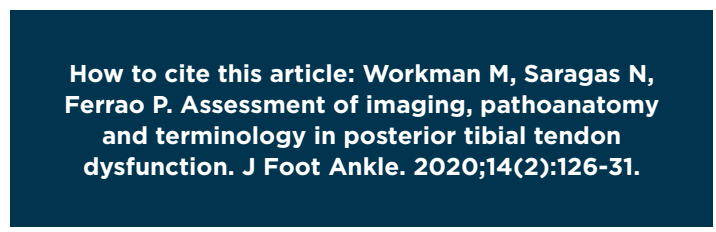


to be up to $100 \%$ sensitive and $88 \%$ specific in detecting tears of the PTT when performed by a competent musculoskeletal ultrasonographer ${ }^{(7)}$. It also allows for dynamic assessment of the PTT in the area of tenderness and can identify possible differentials such as gout. It is an inexpensive and easily accessible modality ${ }^{(8)}$. MRI allows visualisation of the entire ankle and foot, which can help identify other pathologies around the ankle joint. It can identify fatty degeneration and muscle atrophy in cases of complete PTT rupture ${ }^{(9)}$. Oedema within the bone can also be appreciated ${ }^{(10-12)}$. MRI should be considered for patients with inconclusive US examinations and to look for other possible signs of PTTD ${ }^{(9,13)}$.

Historically, it was believed that a tendinitis or tenosynovitis led to PTTD. Trevino et al., in 1981, were the first to describe the histopathology of PTTD. They identified a change from normal parallel collagen bundles to a wavy, loose configuration. Later studies reported that changes consist of mucoid degeneration, neovascularisation, and tendon sheath hypertrophy. This was hypothesised to eventually lead to tendon rupture ${ }^{(14,15)}$. Delmi et al. ${ }^{(16)}$, in 1995, described a tenosynovitis associated with myxoid degeneration, disorganised collagen bundles and calcific deposits. Mosier et al. ${ }^{(17)}$ described a degenerative tendinosis of the PTT, characterised by an excess of mucin deposition, a lack of inflammatory cells, neovascularisation and fibroblast hypercellularity ${ }^{(18)}$. These reports support a degenerative process within the tendon rather than inflammatory changes.

Maffulli et al. ${ }^{(19)}$ suggested using the terms "tendinosis", "paratendinitis" and "tendinitis" when describing general tendon pathology based on histological examination. They proposed using the term "tendinopathy" to describe the clinical syndrome of pain, swelling and reduced performance. Van Dijk et al. ${ }^{(20)}$ noted that the terminology for Achilles tendon pathology had become inconsistent and confusing and that clear terminology was necessary, especially for research purposes. The authors similarly felt that the terminology currently used in describing the pathology of PTTD is vague and needed to be clarified.

This study aimed, by correlating preoperative imaging and intraoperative findings with histological samples, to determine the pathological change that may occur in the posterior tibial tendon of patients undergoing surgery for PTTD and to determine the most appropriate investigations for diagnosis. The secondary aim was to clarify terminology used in the description of this tendon pathology so as to improve the descriptive terminology used when researching, assessing and treating PTTD.

\section{Methods}

Approval for this research was obtained from the institution's ethics and research committee. Records of patients with AAFD who had undergone surgery for stage 2 PTTD correction between January 2016 and January 2020, at a private clinic, were retrospectively reviewed. All cases in which preoperative diagnostic imaging (US or MRI), complete surgical records and histological evaluation of the PTT had been performed were included. Ultrasound is our preferred investigation for PTTD as it can be done dynamically and is inexpensive; MRI is reserved for complex cases with suspected associated pathologies. Cases were excluded if their records were incomplete or data were missing. Seven cases were excluded due to a lack of preoperative imaging. Patient demographics including age, sex and comorbidities were recorded. US, MRI, and surgical findings, as well as histopathological reports, were evaluated. At the time of surgery, specimens of the PTT were obtained, placed in formalin and sent to the anatomical pathology service for microscopic evaluation. All samples were processed at the same laboratory. Data were analysed qualitatively, using descriptive statistics. We reported categorical data in tables with frequencies and percentages and tested normality of the data qualitatively.

\section{Results}

Nineteen patients met the inclusion criteria and were included in the study. Of these, 14 had US examinations and 5 had MRI performed pre-operatively. There were 16 females and 3 males, with a mean age of 59 (range, 45 to 68 ) years. Patient comorbidities included hypertension ( 5 patients), diabetes mellitus ( 4 patients), hypercholesterolaemia ( 3 patients), and thyroid dysfunction (2 patients) (Table 1).

The 14 preoperative US investigations reported moderate to marked degenerative changes/tendinopathy in all cases (Table 2). Further details, including the size and number of longitudinal intratendinous tears and the degree of cross-sectional tendon involvement and fraying, were documented. These changes were noted predominantly at the level of the medial malleolus or distal to it. Fluid was documented within the PTT sheath, more so proximal to the medial malleolus. In all cases, synovial sheath inflammation (synovitis) was also reported.

Five MRI scans were performed. All documented evidence of split tears within the PTT substance with varying degrees of tendon degeneration. Two cases were reported as complete tendon rupture noted proximal to the medial malleolus. Intraoperatively, only one of the two was confirmed to be ruptured. The other had a scarred-down, attenuated tendon.

Intraoperatively, all tendons were thickened and showed gross abnormality with dull, greyish-white discolouration rather than the normal, glistening white appearance, as well as disorganisation of the tendon fibres (Figure 1). Macroscopically, the surrounding sheath appeared reactive, with synovitis, and fluid was expelled when the sheath was incised.

Table 1. Comorbidities

\begin{tabular}{lc}
\hline Comorbidity & Number \\
\hline Hypertension & 5 \\
Diabetes & 4 \\
Hypercholesterolaemia & 3 \\
Depression & 2 \\
Thyroid dysfunction & 2 \\
\hline
\end{tabular}


Table 2. Imaging and histology results

\section{Case}

USS: Degenerate PTT. No defined tear. Severe hypertrophic synovitis with increased surrounding fluid

USS: Tendinopathy throughout tendon. Tear in the distal tendon. Tendon is double its normal size at the malleolus SS: Severe tendinosis of the PTT with high grade distal intrasubstance tears and surrounding granulation tissue

$4 \quad$ USS: Degenerative changes with hypoechoic tendon occupying $30 \%$ of the total cross sectional area

5 USS: Extensive tendinopathy. Small intratendinous tears. Surrounding fluid in keeping with tenosynovitis

6 USS: Tendinopathy distal to MM. Small tear in the region. Minimal increased vascularity

$7 \quad$ USS: Severe degeneration distally with softening and fraying. Synovial hypertrophy. Fluid proximal to malleolus.

$8 \quad$ USS: Inflammation proximal to malleolus. Distally, there is a tendon defect of approx. $24 \%$ of tendon cross sectional area

9 USS: Inflammation of the synovial sheath Small longitudinal intratendon tear just proximal to the navicular

10 USS: Degenerative tendon changes. Tendinopathy at level of MM involves $>50 \%$ cross-sectional area

$11 \quad$ USS: Degenerative changes. Marked tenosynovitis present with fluid collection and inflamed synovium

12 USS: Tendinous disrepair distal to malleolus with inflammatory response. Moderate amount of fluid proximal to the malleolus

13 USS: Tear at malleolus. Degeneration involving 60\% cross sectional area. Fluid within the sheath with marked hypertrophic synovium

$14 \quad$ USS: Tendinopathy at the level of the MM involving $60 \%$ of tendon. Surrounding fluid in keeping with tenosynovitis

15 MRI: Rupture $2.5 \mathrm{~cm}$ above malleolus. Partial tear at the musculotendinous junction. Retraction and thickening of proximal and distal ends

16 MRI: Rupture $\pm 2 \mathrm{~cm}$ above the MM. Retraction and thickening of proximal and distal ends

17 MRI: Acute on chronic tendinitis/tenosynovitis. Underlying interstitial tear of tendon

18 MRI: confirms PTTD with inflammation and a long split in the PTT

\section{Histology findings}

Chronic degenerative changes in the form of oedema, polypoid change and fibrinoid degeneration. Synovium shows neovascularisation. TENDONITIS

Neovascularisation and focal accumulation of ground substance. No active inflammation. NON-SPECIFIC REACTIVE TENDONITIS

Neovascularisation within visceral synovium and tendon with reactive fibrosis evident. No active inflammation. REACTIVE TENDONITIS

Degree of pallor and neovascularisation. No active inflammation. NON-SPECIFIC REACTIVE TENDONITIS

Neovascularisation. Areas of reactive fibroblastic proliferation within tendon No active inflammatory process. NON-SPECIFIC REATIVE TENDONITIS

Inflammation and neovascularisation. No acute inflammation. MILD INFLAMMATION/CHRONIC TENDINITIS

Focal neovascularisation of the tendinous tissue. No active inflammatory process present. NON-SPECIFIC REATIVE SYNOVITIS

Neovascularisation of the visceral synovium.

No significant degenerative/inflammatory changes of tendon. NON-SPECIFIC REACTIVE TENDONITIS

Chronic inflammatory cell infiltrate accompanied by neovascularisation. MILD CHRONIC INFLAMMATION

Neovascularisation within the visceral synovium. No active inflammation REACTIVE TENDONITIS

Eosinophilic degeneration with chronic inflammatory cell infiltrate with neovascularisation. No active inflammation.

Vascularization in the visceral synovium with increased cellularity in tendon. No inflammatory process. NON-SPECIFIC REACTIVE TENDONITIS

Fibrosis and neovascularisation with increased cellularity within tendon. No active inflammatory process. NON-SPECIFIC REACTIVE TENDONITIS

Reactive vascular changes within visceral synovium. No specific degenerative changes in the tendon. BENIGN NON-SPECIFIC REACTIVE SYNOVITIS

Degeneration of central collagen of tendon with a shredded appearance and neovascularisation. No active inflammation. TENDONITIS WITH MILD SYNOVITIS

Non-specific reactive synovitis of the visceral synovium. No active inflammation. REACTIVE TENDONITIS.

Neovascularisation and fibrosis within visceral synovium extending into tendon. No active inflammatory process.

Neovascularisation within visceral synovium extending to the tendon where there is increased fibroblastic activity. No acute or chronic inflammation. NON-SPECIFIC REACTIVE TENDONITIS

Focal areas of eosinophilic degeneration of the collagen. Associated mild synovitis. CHRONIC NON-SPECIFIC TENDONITIS \& SYNOVITIS

MM: Medial malleolus; PTT: posterior tibial tendon; PTTD: posterior tibial tendon dysfunction; USS: ultrasound scan, MRI: magnetic resonance imaging
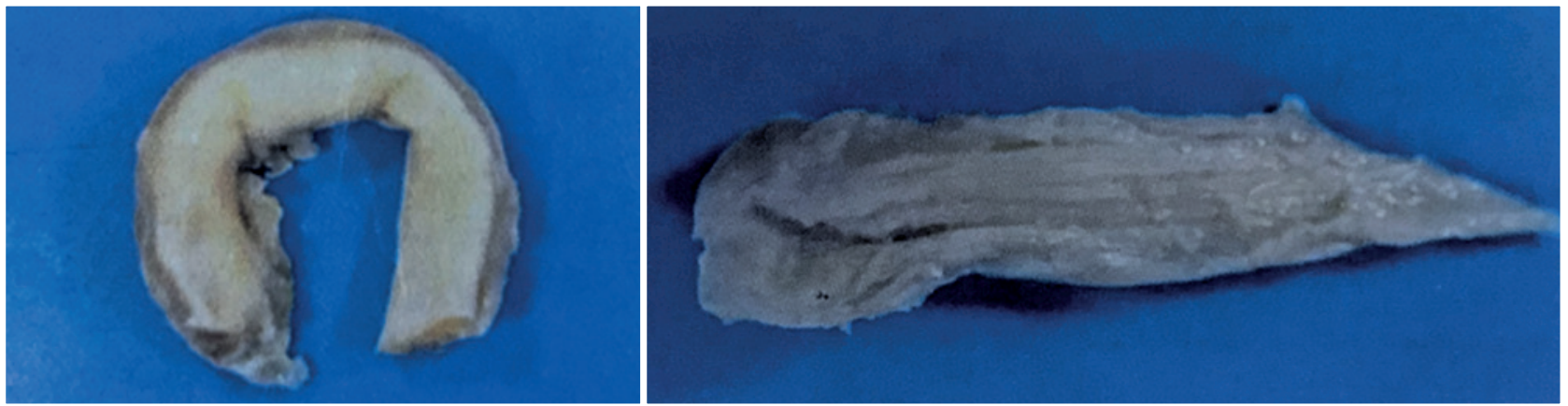

Figure 1. Examples of the gross appearance of the excised posterior tibialis tendon. 
Microscopically, no acute inflammation or granulomatous inflammation was noted within any of the tendon specimens. Sixteen were documented to have non-specific reactive changes within the visceral synovium, in the form of neovascularisation. The remaining 3 cases had documented chronic inflammation and synovitis, but neovascularisation was not specifically mentioned. Changes within the tendon ranged from the description of a "shredded appearance" to reactive fibrosis and fibroblastic proliferation (Figure 2), with increased cellularity and neovascularisation extending into the tendon itself. Most cases were summarised as a "non-specific reactive tendonitis" or "reactive tendonitis" (Table 2 ).

Two cases had histology reports that stated no specific degenerative change noted within the tendon. The US examinations for these 2 cases noted moderate to marked inflammation of the PTT and tendinopathy at the level of the medial malleolus involving $24 \%$ and $60 \%$ of the tendon, respectively. Of these 2 cases, one histology report was concluded as "non-specific reactive synovitis" and the other was summarised as "non-specific reactive tendonitis". The surgical notes in both cases noted synovitis of the tendon sheath with marked tendon thickening and degeneration. Taking into account these 2 cases, correlation of ultrasound with histology findings was $86 \%$ (12/14 cases) for diagnosing marked tendon degeneration/tendinopathy. Although the histology was inconclusive in these 2 cases, the US and intraoperative findings confirmed degeneration of the PTT in all 14 cases. MRI correlated with histology findings in all 5 cases (100\%), but in only $80 \%(4 / 5)$ of cases with intraoperative findings, as the one reported rupture was not present intraoperatively.

\section{Discussion}

The PTT lies posterior to the tibiotalar joint and medial to the subtalar joint. Its primary function is to support the arch and invert the foot. The portion of the PTT that abuts the medial malleolus is characterised by the presence of fibrocartila-

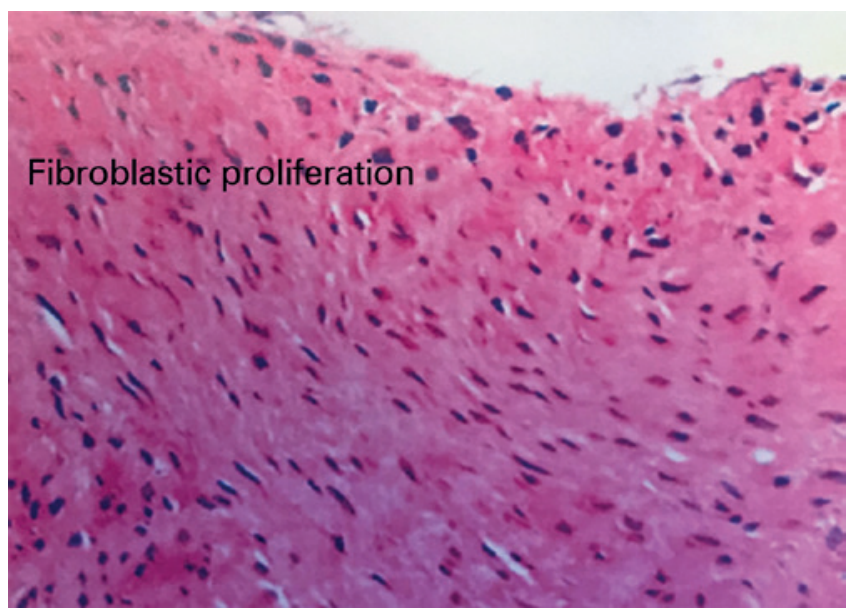

Figure 2. Fibroblastic proliferation noted within the tendon. ge, differentiating it from classic tendon structure. This area is referred to as the gliding tendon ${ }^{(21)}$. The longitudinal intratendinous tears and degree of cross-sectional tendon involvement noted on US and MRI in our study were predominantly at the level of the medial malleolus (in the gliding tendon) or distal to it. This correlates with the hypovascular zone of the tendon but also with an area of increased mechanical shear force on the tendon as it changes course by approximately $80^{\circ}$ at the medial malleolus, suggesting an interplay of factors rather than a single aetiology ${ }^{(10,21)}$.

Previous studies cite $100 \%$ sensitivity with the use of US in the detection of tears within the PTT. It is useful in confirming the diagnosis, but also in ruling out possible differentials. The finding of fluid predominantly proximal to the medial malleolus on US in this study is due to the increased space available for fluid to accumulate as opposed to the area within the tendon sheath at and below the level of the medial malleolus. This fluid was evident at surgery upon incising the sheath, and is in keeping with a paratendinitis. The distal $1-2 \mathrm{~cm}$ of the tendon is not normally surrounded by a tendon sheath, and fluid is normally not identified here ${ }^{(7,22)}$.

Conti et al. ${ }^{(23)}$ reported that MRI may be more accurate than intraoperative visualisation, as an intramural lesion noted on MRI may not be visible macroscopically. The authors concluded that MRI should be considered the gold standard in assessing PTTD. A recent article by Lesiak and Michelson highlighted that MRI is useful in the diagnosis of PTT tears, but that surrounding inflammation can make a normal PTT appear torn. It is hypothesized that surrounding inflammation causes changes in the MRI signal in the PTT that resemble those seen in rupture. For this reason, MRI in the setting of PTT ruptures should be interpreted with caution ${ }^{(11,12)}$. MRI also has the drawback of not being dynamic. In our study, MRI results correlated in all cases (5/5) with histology, but in only $80 \%(4 / 5)$ of cases intraoperatively, as one case reported as being ruptured on MRI was found intact intraoperatively. Arnoldner et al. ${ }^{(9)}$ compared US with 3T MRI in the diagnosis of PTTD. Nine intraoperative diagnoses of PTTD were compared to preoperative US and MRI. The authors concluded that US is slightly more accurate and should be used as the initial diagnostic tool, as it is easily available, cost-effective and visualises the PTT clearly. These findings were in keeping with our results.

Intraoperatively, all 14 specimens correlated with US findings and showed macroscopic evidence of synovitis of the tendon sheath and chronic degenerative tendon change $(100 \%$ correlation). Correlation of US with histology findings was $86 \%$ (12/14 cases) in diagnosing marked tendon degeneration/tendinopathy. Two cases were reported as being normal histologically, but with surrounding mild visceral neovascularisation. The corresponding US described a tendinopathy and a degree of tendon degeneration. Possibly the section of tendon examined histologically was not the section described on preoperative imaging.

The histological description of increased cellularity and neovascularisation within the visceral synovium and the tendon 
indicates an attempt at repair ${ }^{(17)}$. No tendinous inflammation was noted to be present in any of the cases in our series. This has been previously documented in similar studies examining the histology of the Achilles tendon and in ACL injuries ${ }^{(24-26)}$. As hypothesised by Fowble et al. ${ }^{(27)}$, the process is most likely due to overuse and stretching of the posterior tibial tendon, which activates tenocytes of the synovial tendon lining. This results in an angiogenic response and neovascularisation, as was noted in all our cases presenting as paratendinitis. Regarding surgical options for these patients, future histological studies might examine for the presence of mechanoreceptors within the tendon, its sheath, and the distal stump. This may indicate a proprioceptive function, much as it does in the $A C L{ }^{(23)}$, supporting preservation of at least part of the damaged posterior tibial tendon, or its stump, in the reconstruction of AAFD. By maintaining these mechanoreceptors, patients may rehabilitate faster.

All specimens in this study were taken at the time of surgery and therefore represented tendons in an advanced disease stage, having gone through cycles of injury, repair, and remodelling. This may explain why no inflammation was found to be present in these study specimens, but rather evidence of attempted repair. Degeneration of the tendon begins well before clinical presentation of the patient ${ }^{(21,28)}$. A recent study by Klatte-Schutz et al. ${ }^{(26)}$ examined acute versus chronic Achilles tendon injuries. In the study group of acute and chronic ruptures and tendinopathies, the authors detected an inflammatory infiltrate using immunohistochemical examination. These were noted to be significantly lower in the chronic rupture group when compared to the acute ruptures. Since PTTD is a chronic disorder, this would support our findings of no inflammation in the histological specimens. Immunohistochemical examination does appears to offer a more precise histological diagnosis. Further studies are needed to investigate possible genetic predisposition to tendon degeneration, particularly examining the collagen and matrix remodelling gene pathways in individuals. Histology is useful in cases of suspected inflammatory arthritis, which is commonly associated with PTTD. This is typically supported by the presence of characteristic pathological findings in synovial tissue. However, no single histological feature is diagnostic ${ }^{(29)}$.

Recent literature has made a concerted effort to avoid the blanket term "tendonitis" when referring to diseased tendons. This has led to a confusing expansion in the descriptive vocabulary used, with terms such as tendonitis, tendinosis, peritendinitis, paratendinitis and paratendinopathy being used somewhat interchangeably to provide more detailed depictions of the underlying processes. According to the MedTerms Medical Dictionary (<www.medicinenet.com>), peri- is a prefix meaning around or about. Para- is also a prefix with many meanings, including alongside, beside, near or resembling. -osis is a suffix denoting a process, condition, or state, usually abnormal or diseased. -opathy is a suffix meaning a disease or disorder. The suffix -itis denotes an inflammatory disease process ${ }^{(30)}$.

It follows that tendonitis is the inflammation of a tendon. Tendinosis is the preferred term for degenerative changes purely within a tendon without an identified inflammatory component. Peritendinitis and paratendinitis denote an inflammatory process involving the surrounding or nearby tendon sheath, and are not necessarily associated with an intratendinous process. These terms are all dependent on operative confirmation of the condition. Puddu et al.(31), in their study of Achilles tendon pathology, surgically identified cases with only peritendinous inflammation, while others showed degeneration of the tendon itself in addition to peritendon involvement. They suggested the term "peritendinitis" for this type of inflammation and distinguished an isolated peritendinitis from a combination of peritendinitis with tendinosis. The current literature defines PTTD as a tendinosis with no inflammation(21,31). All our cases had both synovitis of the tendon sheath and tendinosis of the PTT. As supported by the findings in this study of PTTD, the correct nomenclature, based on the clinical presentation and preoperative imaging, would be that of a pantendinopathy, which was confirmed on the surgical and histological findings of a peritendinitis with tendinosis. We believe "pantendinopathy" is a better description of the pathological process in PTTD, rather than simply calling it a tendinosis.

Strengths of this study include the availability of preoperative imaging, intraoperative findings and histology results, allowing for a chronological examination of the disease process and clear correlation of investigations with findings. Limitations include the retrospective nature of the study and relatively small sample size.

\section{Conclusion}

This study confirms clear histological degenerative changes within the substance of the posterior tibial tendon together with inflammation of the paratenon, found on preoperative imaging and intraoperative findings, in patients who undergo corrective surgery for PTTD. These findings are in keeping with a pantendinopathy, i.e., peritendinitis with tendinosis. We suggest using the term "pantendinopathy" when describing the pathological changes present in PTTD. Preoperative ultrasound imaging and intraoperative confirmation of PTTD is accurate; histological confirmation is unnecessary.

Authors' contributions: Each author contributed individually and significantly to the development of this article: MW *( https://orcid.org/O000-00027999-9069) performed bibliographic review, survey of the medical records, formatting of the article and approved the final version; NS *( https://orcid. org/0000-0002-5566-7588) conceived and planned the activities that led to the study, performed the surgeries and data collection and approved the final version; PF * (https://orcid.org/0000-0003-4639-0326) interpreted the results of the study, formatted the article, participated in the review process and approved the final version. *ORCID (Open Researcher and Contributor ID) iD. 


\section{References}

1. Dyal C, Feder J, Deland DT, Thompson FM. Pes planus in patients with posterior tibial tendon insufficiency: asymptomatic versus symptomatic foot. Foot Ankle Int.1997;18(2): 85-8.

2. Gazdag AR, Cracchiolo, A. Rupture of the posterior tibial tendon. evaluation of injury of the spring ligament and clinical assessment of tendon transfer and ligament repair. J Bone Joint Surg Am. 1997;79(5): 675-81.

3. Miller MD, Thompson SR, Hart JA, editors. Review of orthopaedics $6^{\text {th }}$ ed. Philadelphia: Elsevier Saunders; 2012. p. 478-83.

4. Johnson KA, Strom DE. Tibialis posterior tendon dysfunction. Clin Orthop Relat Res. 1989 Feb;(239):196-206.

5. Myerson MS. Adult acquired flatfoot deformity: treatment of dysfunction of the posterior tibial tendon. Instr Course Lect. 1997; 46:393-405.

6. Myerson MS, Badekas A, Schon LC. (2004). Treatment of stage II posterior tibial tendon deficiency with flexor digitorum longus tendon transfer and calcaneal osteotomy. Foot Ankle Int. 2004;25(7):445-50.

7. Van Holsbeeck MT, Introcaso J. Musculoskeletal ultrasound. $3^{\text {rd }}$ ed. Philadelphia: Jaypee Brothers; 2016

8. Albano D, Martinelli N, Bianchi A, Romeo G, Bulfamante G, Galia $M$, et al. Posterior tibial tendon dysfunction: clinical and magnetic resonance imaging findings having histology as reference standard. Eur J Radiol. 2018;99:55-61.

9. Arnoldner MA, Gruber M, Syre S, Kristen KH, Trnka HJ, Kainberger $F$, et al. Imaging of posterior tibial tendon dysfunctioncomparison of high-resolution ultrasound and 3T MRI. Eur J Radiol. 2015;84(9):1777-81.

10. Petersen W, Hohmann G, Pufe T, Tsokos M, Zantop T, Paulsen F, et al. Structure of the human tibialis posterior tendon. Arch Orthop Trauma Surg. 2004;124(4): 237-42.

11. Soliman SB, Spicer PJ, Van Holsbeeck, MT. Sonographic and radiographic findings of posterior tibial tendon dysfunction: a practical step forward. Skelet Radiol. 2019;48(1):11-27.

12. Lesiak AC, Michelson JD. Posterior tibial tendon dysfunction: imperfect specificity of magnetic resonance imaging. Foot Ankle Surg. 2020;26(2):224-27.

13. Snell RS. Clinical anatomy. $7^{\text {th }}$ ed. Baltimore: Lippincott Williams \& Wilkins; 2004

14. Trevino S, Gould N, Korson R. Surgical treatment of stenosing tenosynovitis at the ankle. Foot Ankle Int. 1981 2(1):37-45

15. Sharma P, Maffulli N. Biology of tendon injury: healing, modeling and remodeling. J Musculoskelet Neuronal Interact. 2006;6(2):181-90.
16. Delmi M, Kurt AM, Meyer JM. Calcification of the tibialis posterior tendon: a case report and literature review. Foot Ankle Int 1995;16(12): 792-5.

17. Mosier SM, Dosier DR, Pomeroy GC, Manoli A. Pathology of the posterior tibial tendon in posterior tibial tendon insufficiency. Foot Ankle Int. 1998;19(8):520-4.

18. Goncalves-Neto J, Witzel SS, Teodoro WR, Carvalho-Junior $A E$, Fernandes TD, Yoshinari $\mathrm{HH}$. Changes in collagen matrix composition in human posterior tendon dysfunction. Joint Bone Spine. 2002;69(2): 189-94.

19. Maffulli N, Khan KM, Puddu G. Overuse Tendon conditions: time to change a confusing terminology. Arthroscopy. 1998; 14(8):840-3.

20. Van Dijk CN, Van Sterkenburg MN, Wiegerinck JI, Karlsson J, Maffulli, N. Terminology for achilles tendon related disorders. Knee Surg Sports Traumatol Arthrosc. 2011;19(5):835-41.

21. Guelfi M, Pantalone A, Mirapeix RM, Vanni D, Usuelli FG, Salini V. Anatomy, pathophysiology and classification of posterior tibial tendon dysfunction. Eur Rev Med Pharmacol Sci. 2017;21(1):13-9.

22. Lhoste-Trouilloud A. The tibialis posterior tendon. J. Ultrasound 2012;15(1):2-6.

23. Conti, S, Michelson J, Jahss M. Clinical significance of magnetic resonance imaging in preoperative planning for reconstruction of posterior tibial tendon ruptures. Foot Ankle Int. 1992;13(4):208-14

24. Sonnery-Cottet B, Bazille C, Hulet C, Colombet P, Cucurulo T, Panisset JC, et al. Histological features of the $A C L$ remnant in partial tears. Knee. 2014;21(6):1009-13.

25. Maffulli N, Kader D. Tendinopathy of tendo achillis. J Bone Joint Surg Br. 2002;84(1):1-8.

26. Klatte-Schutz F, Minkwitz S, Schmok A, Bormann N, Kurtoglu A, Tsitsilonis S, et al. Different achilles tendon pathologies show distinct histological and molecular characteristics. Int $\mathrm{J}$ Mol Sci. 2018;19(2):404

27. Fowble VA, Vigorita VJ, Bryk E, Sands AK. Neovascularity in Chronic posterior tibial tendon insufficiency. Clin Orthop Relat Res. 2006;450:225-30.

28. Scott A, Beckman L, Speed C. Tendinopathy - update on pathophysiology. J Orthop Sport Phys Ther. 2015;45(11):833-41.

29. Pitzalis C, Kelly S, Humby F. New learnings on the pathophysiology of RA from synovial biopsies. Curr Opin Rheumatol. 2013;25(3):334-44

30. MedicineNet. [cited 2020 Apr. 30]. Available from: https://www. medicinenet.com/script/main/hp.asp.

31. Puddu G, Ippolito E, Postaccini F. A classification of achilles tendon disease. Am J Sports Med. 1976;4(4):145-50. 